\title{
The Results of Treating Failed Back Surgery Syndrome by Adhesiolysis: Comparing the One- and Three-Day Protocols
}

\author{
Behnam Hossieni,, ${ }^{1}$ Payman Dadkhah, ${ }^{1}$ Siamak Moradi, ${ }^{1}$ Seyed Masoud Hashemi, ${ }^{1, *}$ and Farshad \\ Safdari $^{2}$ \\ ${ }^{1}$ Clinical Research Development Unit, Shohada Tajrish Hospital, Shahid Beheshti University of Medical Sciences, Tehran, Iran \\ ${ }^{2}$ Orthotist and Prosthetist, Bone Joint and Related Tissues Research Center, Shahid Beheshti University of Medical Sciences, Tehran, Iran \\ "Corresponding author: Dr. Seyed Masoud Hashemi, Akhtar Hospital, Elahieh, Tehran, Iran. Tel: +98-2188444408, E-mail: hashemimasoud@gmail.com
}

Received 2016 June 22; Revised 2017 February 13; Accepted 2017 April 25.

\begin{abstract}
Background: The optimal treatment of failed back surgery syndrome (FBSS) is controversial. Limited studies have demonstrated the satisfactory outcomes of percutaneous adhesiolysis in FBSS, which can be performed as a 1 day or 3 days procedure. In the current randomized clinical trial, we compared the clinical and functional outcomes of these 2 techniques.

Methods: In this study, 60 patients with FBSS were randomly assigned into 2 equal groups: 1 day group and 3 days group. Before and at 4 and 12 weeks after the procedure, pain intensity was measured using visual analogue scale (VAS). The Oswestry disability index (ODI) was also completed. Pain reduction of $50 \%$ or more was defined as treatment success.

Results: Significant pain relief and ODI improvement were obtained in the 2 groups with adhesiolysis $(\mathrm{P}<0.001)$. However, pain intensity remained the same before and at 4 and 12 weeks after adhesiolysis. ODI score was significantly lower in 1 day group in the 1 month visit $(\mathrm{P}<0.001)$. Treatment was successful in $76.7 \%$ and $83.3 \%$ of the patients in 1 day and 3 days groups, respectively $(\mathrm{P}=$ 0.519).

Conclusions: Adhesiolysis is an effective treatment for pain relief and functional improvement in FBSS. The results of 1 day and 3 days procedures are comparable. Based on these findings, the authors recommend using 1 day technique, which can potentially decrease the patients' discomfort, hospital stay, and cost of treatment.
\end{abstract}

Keywords: Adhesiolysis, Failed Back Surgery Syndrome, Epidural Scar

\section{Background}

Along with desirable results of surgical treatment of various spine problems, residual constant pain after surgery is one of the most important complications of the treatments. Despite advances achieved in the field of spinal surgery, the failure rate of these therapeutic methods has not been decreased in the last 2 decades $(1,2)$. It has been reported that among 300,000 to 400,000 surgeries annually done to relieve chronic low back pain (LBP), only $50 \%$ to $60 \%$ are successful (3). In fact, failed back surgery syndrome (FBSS) or postsurgery syndrome does not have a precise definition; FBSS is a set of syndromes, pain, and disability after spinal surgery. In various studies, the incidence of FBSS has been reported to be $10 \%$ to $40 \%(2,4)$.

Several causes have been introduced for FBSS including pressure on the nerve root due to disk reherniation or retained disk fragment, epidural fibrosis, acquired stenosis, and segmental instability. However, about 20\% to $36 \%$ of FBSS occur due to epidural fibrosis, which is a progressive disease $(2,4)$.

FBSS treatment methods vary and include conserva- tive treatments, oral medications, combination of topical agents, epidural injection of different drugs, trigger point injection, percutaneous adhesiolysis, high-dose oxycodone/naloxone, spinal endoscopic adhesiolysis, facet joints nerve block, Sacrococcygeal gap injection, sacroiliac joint injection, spinal cord stimulation (SCS), placement of intratecal catheter, and resurgery that although have been successful to some extent, they have some defects and limitations (4-26).

Percutaneous adhesiolysis is a relatively new technique, which wasintroduced about 25 years ago for the treatment of FBSS (12). However, few studies have been conducted in this field. In this method, undesirable and destructive effects and scar formation are mechanically disappeared, which can lead to decompression of nerve roots and pain relief. In addition, percutaneous epidural adhesiolysis leads to entering large amounts of medicine with high concentration to the desired location. However, the results of previous studies have been very different, and success rates varied between $48.7 \%$ to $73 \%(4,8,10)$. Two main approaches for performing percutaneous adhesiolysis include 1 day and 3 days methods. To our knowledge, no 
study has compared the results and complications of these 2 methods. In this study, we compared the effectiveness of 1 and 3 days epidural adhesiolysis methods in the treatment of FBSS. As no difference was found between the 2 methods, we can prevent patients' hospitalization and increase health care costs.

\section{Methods}

In this randomized clinical trial, 60 patients with FBSS, who referred to our pain clinic at 2014, were investigated. Diagnosis of epidural adhesion was made based on the clinical findings and MR imaging. Clinical findings may include increased back pain during walking or sitting, referral pain, muscular spasm, hip or sacroiliac joint pain, headache, burning, electrical shock-like pain, numbness and weakness, with symptoms indicating the alteration of proprioception such as dizziness or tinnitus and dysfunction of bladder, bowel, and autonomic system. Presence of some of these findings and history of a previous spinal surgery, in addition to abnormal MR images, were considered as the criteria for diagnosis of FBSS. Inclusion criteria included age over 18 years, and at least 6 months pain history with or without radicular pain after spinal surgery. Exclusion criteria included suspected facet joint pain, any epidural injection, or other invasive therapeutic methods within the last 6 months. Other exclusion criteria were pregnancy, breastfeeding, addiction, and mental problems. All patients were asked to sign a written informed consent.

After registering demographic information, pain intensity was measured using visual analogue scale (VAS), and functional status was investigated using Oswestry disability index (ODI) questionnaire. Patients were assigned randomly into 2 groups of 1 day or 3 days technique using random number table.

In 1 day group, after preoperative evaluation, antibiotic was administered via IV access based on the protocol and patient condition. The procedure was performed in prone position. Sedation was administered gently. The beam of C-arm was adjusted over the lumbosacral spine. An epidural needle gauge 18 was inserted in caudal epidural space at first. Then, through the needle, $2-5$ cc of Omnipaque 240 was injected to detect filling the defect site using lumbar or caudal epidurugram. After detecting the lesion site, a spring- guided Racz catheter was gently inserted through the RK needle into the filling defect area using flouroscopy and findings of the physical examination. Thereafter, 10 to $20 \mathrm{cc}$ of saline with hyaluronidase was injected through a catheter washout. Then, 5 cc of lidocaine $2 \%$ was kept in site as a local injection. If epidural or nerve root filling were observed, complete adhesiolysis would have been obtained.
The catheter was closed using bio-occlusive dressing. In recovery, after resolving the motor block, 15 to 30 minutes after local anesthetic injection (10 cc of bupivacaine $0.25 \%$ ), $6 \mathrm{cc}$ of saline $5 \%$, with or without hyaluronidase, was injected in 2 doses of $3 \mathrm{cc}$. In the next step, 40 to $80 \mathrm{mg}$ of alcohol-free triamcinolone was injected. Finally, by injecting $0.5-1 \mathrm{cc}$ of normal saline, the catheter was removed. Motor and sensory function was evaluated for all of the patients. Finally, IV access was removed and the patient was discharged.

After inserting an epidural needle gauge, 18 caudal epidural space, $10 \mathrm{~mL}$ of Omnipaque 240 was injected for the 3 day group. After placement of Racz catheter, saline with hyaluronidase was injected, and then, bupivacaine $0.25 \%$ combined with $40 \mathrm{mg}$ of triamcinolone were injected. In recovery, $9 \mathrm{cc}$ of saline $5 \%$ was injected half an hour after steroid-anesthetic injection. After the procedure was complete, the catheter was secured using 2 - 0 nylon with application of antibiotic ointment. Then, the patient was transferred to the ward and on the third day, $10 \mathrm{cc}$ of bupivacaine $0.25 \%$ was injected after negative aspiration within half an hour, followed by injection of $10 \mathrm{cc}$ of saline 5\%. Finally, the catheter was removed and a triple antibiotic ointment was applied on the wound. The most involved level was targeted for adhesiolysis in the 2 groups.

The patients were asked to attend the clinic for reexamination 4 and 12 weeks after discharge. In these visits, pain intensity and functional status were assessed by VAS and ODI, respectively. In this study, pain reduction of 50\% or more was considered as treatment success. Finally, collected data were statistically analyzed.

Quantitative data were presented as mean \pm standard deviation, and qualitative data were presented as number and percentage. To compare pre- and post-treatment, VAS and ODI in each group, paired $t$ test or Wilcoxon test was used, and to compare the 2 groups, independent $t$ test or Mann-Whitney U test was used. All analyses were performed using statistical software SPSS version 16. In this study, $\mathrm{P}<0.05$ was considered significant.

\section{Results}

Demographic information of the 2 groups is demonstrated in Table 1 , showing no statistically significant differences between the groups in age, gender, BMI, and spinal involvement level. Pain intensity and ODI score were compared in Table 2. As demonstrated, pain intensity before and after treatment was the same in the 2 groups. Also, the ODI score was the same before the treatment and in the final visit in the 2 groups, while ODI was significantly lower in 1 day group in the 1 month visit, $(\mathrm{P}<0.001)$. In addition, ODI score in the final visit was lower in 1 day group than 
the 3 days group, but the difference was not statistically significant. In the final visit, pain intensify and ODI score in both groups improved significantly compared to before the treatment $(\mathrm{P}<0.001)$. By definition of the success of treatment (reduction in pain intensity by $50 \%$ or more), adhesiolysis was successful in 23 (76.7\%) and 25 (83.3\%) patients in 1 day and 3 days groups, respectively. However, this difference was not statistically significant $(P=0.519)$.

Table 1. Comparison of Demographic Data

\begin{tabular}{|cccc}
\hline Group & $\begin{array}{c}\text { One-Day Group }(\mathbf{n} \\
=\mathbf{3 0})\end{array}$ & $\begin{array}{c}\text { Three-Dayss } \\
\text { Group }(\mathbf{n}=\mathbf{3 0})\end{array}$ & P Value \\
\hline Age, $\mathbf{y}$ & $61.3 \pm 7.5$ & $62.2 \pm 6.8$ & 0.629 \\
\hline Gender & $(44-74)$ & $(47 / 76)$ & \\
\hline Male & 18 & 16 & 0.602 \\
\hline Female & 12 & 14 & \\
\hline BMI, kg/m & $25.78 \pm 2.36$ & $26.17 \pm 2.27$ & 0.639 \\
\hline $\begin{array}{c}\text { Level of } \\
\text { involvement }\end{array}$ & $(21.34-30.52)$ & $(20.85-31.67)$ & \\
\hline L3-L4 & & & 0.705 \\
\hline L4-L5 & 16 & 11 & \\
\hline L5-S1 & 6 & 5 & \\
\hline
\end{tabular}

\section{Discussion}

The present study revealed that adhesiolysis with both 1 and 3 day techniques can play an important role in relieving pain and improving function in patients with FBSS. In both methods, success rate was satisfactory and comparable, and in some cases it was better than the results of other FBSS treatment methods.

Some authors have clearly shown the relationship between epidural fibrosis formation and radicular pain and clinical results. Ross et al. stated that the presence of a scar tissue around the nerve roots increases the incidence of radicular pain up to 3.2 times (27). Also, in a study by Bokov et al. it was found that the epidural scar tissue formation after microdiscectomy due to the disc extrusion or sequester was the cause of recurrent pain in $12.3 \%$ of patients with FBSS (2). Furthermore, in a randomized clinical trial conducted to evaluate the efficacy of ACON-L gel to prevent scar tissue formation, Maroon et al. found a significant relationship between peridural scar formation and persistent low back pain after discectomy (28). However, the role of epidural fibrosis in the incidence of FBSS is still indeterminate, and some studies have found no relationship between FBSS and the surgery outcome. In a clinical trial, Rönnberg found no significant relationship between the presence of a wide peridural scar or the location of scar formation and the clinical results (29).

However, in recent years, the use of adhesiolysis has become one of the most common methods in the treatment of pain due to FBSS and has been used in many pain clinics around the world. This technique is a minimally invasive technique for the treatment of radicular pain and spinal problems, which is used when conservative or more common methods like epidural injection have failed (30). The main hypothesis for using adhesiolysis in FBSS is that the presence of epidural fibrosis can potentially cause pain and prevent analgesic drugs to reach the affected site based on the findings of the above-mentioned studies $(2,27,28)$. Other suggested mechanisms for adhesiolysis are washing out inflammatory cytokines in the damaged site, lavage of epidural space, reduced ectopic exudates of damaged nerves, and increased blood flow in ischemic nerve roots (30).

One of the most important methods for the treatment of FBSS is spinal cord stimulation, which has been associated with desirable results. Abeloos et al. stated that $75 \%$ of patients with refractory FBSS, who were treated by SCS, were satisfied with the treatment results after 8.3 years (31). Reverberi et al. also reported desirable results for the treatment of FBSS by SCS and peripheral nerve field stimulation in the short-term follow-up (19). In 2 systematic reviews, Frey et al. and Kelly et al. stated that SCS can greatly affect the treatment of FBSS, but more studies are needed (6, 14). Although systematic reviews are the best type of studies to evaluate the efficacy of treatment modalities (32), because SCS is a highly invasive and expensive method, it is preferred to use other techniques before using SCS. In fact, if easier therapeutic methods are not effective, spinal cord stimulation should be performed before deciding on surgery.

Although epidural injection is a relatively simple and common method with fairly good results, it has several limitations. Manchkanti et al. found that in FBSS, caudal epidural injection caused improving functional status in $55 \%$ and pain reduction in $60 \%$ to $70 \%$ of patients (33). In another study, the same authors found that after 1 year, pain relief and functional improvement were only seen in $53 \%$ to $-59 \%$ of patients with FBSS, following epidural injection (17). As mentioned, in cases in which a fibrous tissue is formed, the integrity of the epidural space is lost, and epidural fibrosis can prevent the drug to reach the affected site. Recently, Lee et al. indicated that adhesiolysis, compared to transforaminal epidural steroid injection, is significantly more effective in relieving pain and improving 
Table 2. Comparison of Pain Intensity and ODI

\begin{tabular}{|c|c|c|c|c|}
\hline Group & & One-Day Group $(n=30)$ & Three-Dayss Group $(\mathbf{n}=\mathbf{3 0})$ & PValue \\
\hline \multirow{6}{*}{ Pain (VAS) } & \multirow{2}{*}{ Before the procedure } & $8.2 \pm 1.2$ & $8.6 \pm 0.9$ & \multirow{2}{*}{0.162} \\
\hline & & $(6-10)$ & $(7-10)$ & \\
\hline & \multirow{2}{*}{ One month after the procedure } & $4.8 \pm 1.1$ & $5.3 \pm 1$ & \multirow{2}{*}{0.082} \\
\hline & & $(3-7)$ & $(3-7)$ & \\
\hline & \multirow{2}{*}{ Three months after the procedure } & $3.5 \pm 1.9$ & $3.2 \pm 1.7$ & \multirow{2}{*}{0.517} \\
\hline & & $(1-9)$ & $(1-8)$ & \\
\hline \multirow{6}{*}{ ODI, \% } & \multirow{2}{*}{ Before the procedure } & $75.4 \pm 14$ & $72.2 \pm 9.2$ & \multirow{2}{*}{0.14} \\
\hline & & $(14-68)$ & $(56-88)$ & \\
\hline & \multirow{2}{*}{ One month after the procedure } & $32.7 \pm 13.4$ & $49.7 \pm 10.9$ & \multirow{2}{*}{$<0.001$} \\
\hline & & $(14-68)$ & $(24-66)$ & \\
\hline & \multirow{2}{*}{ Three months after the procedure } & $23.9 \pm 17.8$ & $31.1 \pm 16$ & \multirow{2}{*}{0.103} \\
\hline & & $(4-78)$ & $(6-68)$ & \\
\hline
\end{tabular}

functional in patients with FBSS (34). Some studies have also shown that epidural injection is not highly efficient and it only relieves pain temporarily. Also, Manchikanti et al. in a clinical trial with a one year follow-up revealed that epidural injection and epidural adhesiolysis were successful in $12 \%$ and $73 \%$ of the FBSS patients, respectively (4). In another clinical trial with a 2-year follow-up, the same authors found that pain relief and functional improvement were obtained in $82 \%$ of the patients in 1 day adhesiolysis group and only in $5 \%$ of patients in epidural injection group. On average, each patient underwent epidural injection for $6.4 \pm 2.3$ (35). Also, Chung-Jing et al. demonstrated that after 6 months, the rate of clinical effectiveness in epidural adhesiolysis group was 50\% and it was 5.26\% in dexamethasone injection group (8).

The primary method of adhesiolysis introduced by Racz included a 3-day period with the catheter in the epidural space and with injection of a different drug every day (36). After a while, Manchikanti et al. changed this method and suggested a 1 day outpatient method (37). Theoretically, the 1 day method has many advantages over the 3 days method. In this method, the catheter is removed immediately after drug injection and the patient is discharged. Therefore, the patient experiences less pain and discomfort. Furthermore, the 1 day technique can play a significant role in reducing costs by reducing the hospital stay. However, to our knowledge, the results and efficacy of these 2 techniques of epidural adhesiolysis have not been compared previously. Thus, in this randomized clinical trial, 2 groups of patients with FBSS were treated by 1 and 3 day epidural adhesiolysis. In our study, both techniques effectively reduced pain intensity and improved ODI score without any significant difference. However, the significantly lower 1 month ODI in the 1 day group showed that this technique can be associated with faster functional im- provement. Thus, one should note that although there was no significant difference between the 2 methods, the 3 month ODI score was lower in the 1 day group compared to the 3 day group, and perhaps, if the number of patients was increased in our study, the difference might have reached statistical significance.

\subsection{Conclusions}

Given that the final results of the 2 methods were statistically identical and both protocols had high safety, the authors recommend using the 1 day technique due to the decreased duration of the procedure and hospital stay, which can be associated with less patient discomfort and treatment cost. In addition, it should be noted that ODI score was lower in 1 day group, encouraging the use of this protocol. However, more future randomized studies with larger sample sizes are required.

\section{References}

1. Rahimzadeh P, Sharma V, Imani F, Faiz HR, Ghodraty MR, NikzadJamnani AR, et al. Adjuvant hyaluronidase to epidural steroid improves the quality of analgesia in failed back surgery syndrome: a prospective randomized clinical trial. Pain Physician. 2014;17(1):E7582. [PubMed: 24452659].

2. Bokov A, Isrelov A, Skorodumov A, Aleynik A, Simonov A, Mlyavykh S An analysis of reasons for failed back surgery syndrome and partial results after different types of surgical lumbar nerve root decompression. Pain Physician. 2011;14(6):545-57. [PubMed: 22086096].

3. Hua S, Levy R. In: Essentials of Pain Medicine and Regional Anesthesia. Benyon H, Srinivasa G, Borsook D, Malloy R, Strichartz G, editors. Philadelphia: Churchill Livingstone;1999. Spinal cord stimulation for failed back surgery syndrome.

4. Manchikanti L, Singh V, Cash KA, Pampati V, Datta S. A comparative effectiveness evaluation of percutaneous adhesiolysis and epidural steroid injections in managing lumbar post surgery syndrome: a randomized, equivalence controlled trial. Pain Physician. 2009;12(6):E355-68. [PubMed: 19935992]. 
5. Bujedo BM. Treatment of failed back surgery syndrome in a fortythree-year-old man with high-dose oxycodone/naloxone. Anesth Pain Med. 2015;5(2):e21009. doi: 10.5812/aapm.21009. [PubMed: 25893186].

6. Frey ME, Manchikanti L, Benyamin RM, Schultz DM, Smith HS, Cohen SP. Spinal cord stimulation for patients with failed back surgery syndrome: a systematic review. Pain Physician. 2009;12(2):379-97. [PubMed: 19305486].

7. Chan CW, Peng P. Failed back surgery syndrome. Pain Med. 2011;12(4):577-606. doi: 10.1111/j.1526-4637.2011.01089.x. [PubMed: 21463472].

8. Chun-jing H, Hao-xiong N, jia-xiang N. The application of percutaneous lysis of epidural adhesions in patients with failed back surgery syndrome. Acta Cir Bras. 2012;27(4):357-62. [PubMed: 22534813].

9. Hou JC, Zheng TY, Li DY, Zhi MX. [Sacrococcygeal gap injection for the treatment of failed back surgery syndrome]. Zhongguo Gu Shang. 2014;27(3):229-31. [PubMed: 24974427].

10. Hsu E, Atanelov L, Plunkett AR, Chai N, Chen Y, Cohen SP. Epidural lysis of adhesions for failed back surgery and spinal stenosis: factors associated with treatment outcome. Anesth Analg. 2014;118(1):215-24. doi: 10.1213/ANE.0000000000000042. [PubMed: 24356168].

11. Hussain A, Erdek M. Interventional pain management for failed back surgery syndrome. Pain Pract. 2014;14(1):64-78. doi: 10.1111/papr.12035. [PubMed: 23374545].

12. Jamison DE, Hsu E, Cohen SP. Epidural adhesiolysis: an evidencebased review. J Neurosurg Sci. 2014;58(2):65-76. [PubMed: 24819483].

13. Kat'uch V, Pataky F, Kat'uchova J, Gajdos M, Radonak J. [Surgical management of the failed back surgery syndrome (FBSS) using posterior lumbar interbody fusion (PLIF) with posterior transpedicular stabilization]. Rozhl Chir. 2010;89(7):450-8. [PubMed: 20925263].

14. Kelly GA, Blake C, Power CK, O'Keeffe D, Fullen BM. The impact of spinal cord stimulation on physical function and sleep quality in individuals with failed back surgery syndrome: a systematic review. Eur J Pain. 2012;16(6):793-802. doi: 10.1002/j.1532-2149.2011.00092.x. [PubMed: 22337598].

15. Khosravi MB, Azemati S, Sahmeddini MA. Gabapentin versus naproxen in the management of failed back surgery syndrome; a randomized controlled trial. Acta Anaesthesiol Belg. 2014;65(1):31-7. [PubMed: 24988825].

16. Manchikanti L, Manchikanti KN, Gharibo CG, Kaye AD. Efficacy of Percutaneous Adhesiolysis in the Treatment of Lumbar Post Surgery Syndrome. Anesth Pain Med. 2016;6(2):e26172. doi: 10.5812/aapm.26172v2. [PubMed: 27574583].

17. Manchikanti L, Singh V, Cash KA, Pampati V, Datta S. Management of pain of post lumbar surgery syndrome: one-year results of a randomized, double-blind, active controlled trial of fluoroscopic caudal epidural injections. Pain Physician. 2010;13(6):509-21. [PubMed: 21102963].

18. Taheri A, Khajenasiri AR, Yazdi NN, Safari S, Sadeghi J, Hatami M. Clinical Evaluation of Percutaneous Caudal Epidural Adhesiolysis With the Racz Technique for Low Back Pain Due to Contained Disc Herniation. Anesth Pain Med. 2016;6(3):e26749. doi: 10.5812/aapm.26749.

19. Reverberi C, Dario A, Barolat G. Spinal cord stimulation (SCS) in conjunction with peripheral nerve field stimulation (PNfS) for the treatment of complex pain in failed back surgery syndrome (FBSS). Neuromodulation. 2013;16(1):78-82. doi: 10.1111/j.1525-1403.2012.00497.x. [PubMed: 22985076] discussion 83.

20. Sears NC, Machado AG, Nagel SJ, Deogaonkar M, Stanton-Hicks M, Rezai AR, et al. Long-term outcomes of spinal cord stimulation with paddle leads in the treatment of complex regional pain syndrome and failed back surgery syndrome. Neuromodulation. 2011;14(4):3128. doi: 10.1111/j.1525-1403.2011.00372.x. [PubMed: 21992424] discussion 318.

21. Teixeira MJ, Yeng LT, Garcia OG, Fonoff ET, Paiva WS, Araujo JO. Failed back surgery pain syndrome: therapeutic approach descriptive study in 56 patients. Rev Assoc Med Bras (1992). 2011;57(3):282-7. [PubMed: 21691691].

22. Tomycz ND, Ortiz V, Moossy JJ. Simultaneous intrathecal opioid pump and spinal cord stimulation for pain management: analysis of 11 patients with failed back surgery syndrome. J Pain Palliat Care Pharmacother. 2010;24(4):374-83. doi: 10.3109/15360288.2010.523066. [PubMed: 21133746].

23. Turner JA, Hollingworth W, Comstock BA, Deyo RA. Spinal cord stimulation for failed back surgery syndrome: outcomes in a workers' compensation setting. Pain. 2010;148(1):14-25. doi: 10.1016/j.pain.2009.08.014. [PubMed: 19875232].

24. Waikakul S. Serum 25-hydroxy-calciferol level and failed back surgery syndrome. J Orthop Surg (Hong Kong). 2012;20(1):18-22. doi: 10.1177/230949901202000104. [PubMed: 22535805].

25. Patel VB, Wasserman R, Imani F. Interventional Therapies for Chronic Low Back Pain: A Focused Review (Efficacy and Outcomes). Anesth Pain Med. 2015;5(4):e29716. doi: 10.5812/aapm.29716. [PubMed: 26484298].

26. Safaeian P, Mattie R, Hahn M, Plastaras CT, McCormickZ L, et al. Novel Treatment of Radicular Pain With a Multi-Mechanistic Combination Topical Agent: A Case Series and Literature Review. Anesth Pain Med. 2016;6(2):e33322. doi: 10.5812/aapm.33322.

27. Ross JS, Robertson JT, Frederickson RC, Petrie JL, Obuchowski N, Modic MT, et al. Association between peridural scar and recurrent radicular pain after lumbar discectomy: magnetic resonance evaluation. ADCON-L European Study Group. Neurosurgery. 1996;38(4):855-61. [PubMed: 8692415] discussion 861-3.

28. Maroon JC, Abla A, Bost J. Association between peridural scar and persistent low back pain after lumbar discectomy. Neurol Res. 1999;21 Suppl 1:S43-6. [PubMed: 10214571].

29. Ronnberg K, Lind B, Zoega B, Gadeholt-Gothlin G, Halldin K, Gellerstedt $\mathrm{M}$, et al. Peridural scar and its relation to clinical outcome: a randomised study on surgically treated lumbar disc herniation patients. Eur Spine J. 2008;17(12):1714-20. doi: 10.1007/s00586-008-08058. [PubMed: 18946688].

30. Lee F, Jamison DE, Hurley RW, Cohen SP. Epidural lysis of adhesions. Korean J Pain. 2014;27(1):3-15. doi: 10.3344/kjp.2014.27.1.3. [PubMed: 24478895].

31. Abeloos L, De Witte O, Riquet R, Tuna T, Mathieu N. [Long-term outcome of patients treated with spinal cord stimulation for therapeutically refractory failed back surgery syndrome: a retrospective study]. Neurochirurgie. 2011;57(3):114-9. doi: 10.1016/j.neuchi.2011.07.001. [PubMed: 21907361].

32. Imani F, Rahimzadeh P. Interventional pain management according to evidence-based medicine. Anesth Pain Med. 2012;1(4):235-6. doi: 10.5812/aapm.4514. [PubMed: 24904805].

33. Manchikanti L, Singh V, Cash KA, Pampati V, Datta S. Preliminary results of a randomized, equivalence trial of fluoroscopic caudal epidural injections in managing chronic low back pain: Part 3-Post surgery syndrome. Pain Physician. 2008;11(6):817-31. [PubMed: 19057628].

34. Lee JH, Lee SH. Clinical effectiveness of percutaneous adhesiolysis versus transforaminal epidural steroid injection in patients with postlumbar surgery syndrome. Reg Anesth Pain Med. 2014;39(3):2148. doi: 10.1097/AAP.0000000000000073. [PubMed: 24646624].

35. Manchikanti L, Singh V, Cash KA, Pampati V. Assessment of effectiveness of percutaneous adhesiolysis and caudal epidural injections in managing post lumbar surgery syndrome: 2-year followup of a randomized, controlled trial. J Pain Res. 2012;5:597-608. doi: 10.2147/JPR.S38999. [PubMed: 23293536].

36. Racz GB, Heavner JE, Diede JH. In:. Waldman SD, Winnie AP, editors. Philadelphia: WB Saunders; 1996. pp. 339-51.Lysis of epidural adhesions utilizing the epidural approach. In: Interventional pain management.

37. Manchikanti L, Pakanati RR, Bakhit CE, Pampafi V. Role of Adhesiolysis and Hypertonic Saline Neurolysis in Management of Low Back Pain: Evaluation of Modification of the Racz Protocol. Pain Dig. 1999;9:91-6. 